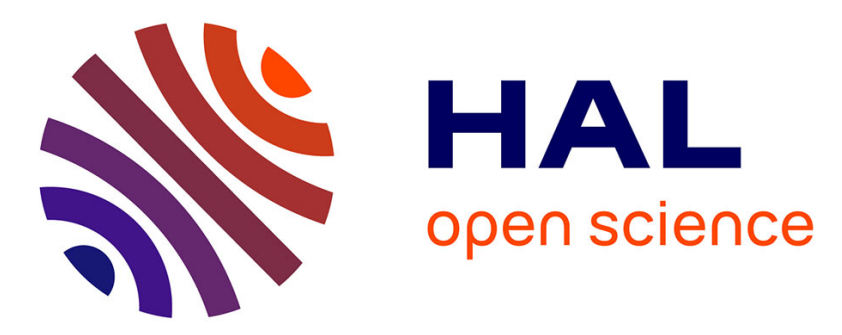

\title{
Loop speed trap data collection method for an accurate short-term traffic flow forecasting
}

Sahraoui Abdelatif, Derdour Makhlouf, Philippe Roose, Djamel Becktache

\section{To cite this version:}

Sahraoui Abdelatif, Derdour Makhlouf, Philippe Roose, Djamel Becktache. Loop speed trap data collection method for an accurate short-term traffic flow forecasting. International Conference on Mobile Web and Intelligent Information Systems, 2016, Vienna, Austria. pp.56-64. hal-02437024

\section{HAL Id: hal-02437024 \\ https://hal-univ-pau.archives-ouvertes.fr/hal-02437024}

Submitted on 13 Jan 2020

HAL is a multi-disciplinary open access archive for the deposit and dissemination of scientific research documents, whether they are published or not. The documents may come from teaching and research institutions in France or abroad, or from public or private research centers.
L'archive ouverte pluridisciplinaire HAL, est destinée au dépôt et à la diffusion de documents scientifiques de niveau recherche, publiés ou non, émanant des établissements d'enseignement et de recherche français ou étrangers, des laboratoires publics ou privés. 


\title{
Loop speed trap data collection method for an accurate short-term traffic flow forecasting
}

\author{
Sahraoui Abdelatif ${ }^{1}$, Derdour Makhlouf ${ }^{2}$, Philippe roose $^{3}$, and Djamel \\ becktache $^{2}$ \\ 1 University of Larbi Tebessi,LAMIS Laboratory, Algeria. \\ s.abdelatif@univ-tebessa.dz \\ 2 University Badji-Mokhtar,LRS Laboratory, Annaba, Algeria \\ \{m.derdour,djamel_bektache\}@yahoo.fr \\ 3 LIUPPA/UPPA, Anglet,2 Allée du Parc Montaury,64600 Anglet, France. \\ Philippe.Roose@iutbayonne.univ-pau.fr
}

\begin{abstract}
despite the growing trend in intelligent transportation systems applications. Besides, there still many problems waiting for an accurate solution such as traffic flow forecasting. In this paper, based on real-time data provided by dual loop speed traps detectors at given slot of time; we propose a cloud data collection method aimed to improve prediction accuracy. To reach this accuracy, two traffic parameters was introduced: average speed and foreseen arrival time between two vehicles. By adopting Choquet integral operator, these parameters can subsequently aggregated to busiest parameters. Afterwards, a simple linear regression is applied for a dual purpose: forecasting and proving that there is a relationship between derived busiest arrival time and the traffic flow $(q)$. Moreover, simulation flowcharts results illustrate that the forecasts by the Choquet operator ensure an accurate results to the real-time data. In contrast, weighted average operator results weak accuracy forecast compared to the real-time data.
\end{abstract}

Keywords: short-term traffic flow forecasting; cloud based-data collection method; Choquet integral operator; weighted average operator; simple linear regression

\section{Introduction}

The short-term and long-term traffic flow prediction horizons have long been considered since each one has concern in a particular period. Regardless to shortterm forecasting horizon, no longer few minutes away for the traffic forecasts, where it has attracted various prediction methods including multivariate time series, kalman filtering method [5], non-parametric regression model [12], artificial neural model [7]. Furthermore, these methodologies can be categorized based on statistical models like linear regression or artificial models like neural models. The predicted arrival time or foreseen arrival time delay and the traffic flow $(q)$, are presents crucial traffic parameters which has attracted great attention in past few years. Whereas, the arrival time can usually use to assess the accuracy prediction 
of the traffic flow [6]. Indeed, prediction accuracy of these parameters can be performed by an effective data collection utilizing road detectors technologies. For traffic parameters forecasting purpose, many works which have been put forward enables aforementioned methods, but an accurate traffic forecasting using data collection has not been seen further. Feng et al [4], propose real-time adaptive traffic control solution based on data collection from connected vehicles. Wherein, a set of multi-linear regression models [8] are devoted for arrival times prediction using data collected by automatic passenger counter (APC). Zhong et al [14], used real-time traffic data were it continuously collected by inductive loop for enabling traffic flow output, while Kalman filtering method takes the advantage of traffic parameters forecasting based on historical models. From other perspective, traffic parameters prediction can be viewed as a complicated process affected by many factors. In which, Bai et al [2], estimates baseline travel times from the historical bus trip data by utilizing Kalman filtering, where it consider three factors as inputs: day time, road segment and weighted average travel time. In this study, we deal with multi-factors influenced traffic flow on which they are in time and space related such as: road segment, safety distance, foreseen arrival time. In particular, the accuracy of the traffic flow is depending on utilized mathematical operator. Bachmann et al [1] uses Choquet fuzzy integral operator to capture a complex relationship between sensors data measurements. Authors in [9] and [13], used weighted average speed in order to calculate the optimal speed estimation. Wherever, we support Choquet integral operator to capture an accurate traffic parameters.

The researches mentioned above treats the accuracy of the traffic parameters and its relationship with predicted vehicle arrival time, where it has not defined so far. For this reason, our contribution is not limited only on demonstrating the correlation between these two variables, but also proven that the foreseen arrival time aggregation factor has a great effect on traffic flow prediction.

In this paper, using dual loop speed traps detectors, we propose a cloud servicebased data collection method aimed to add accuracy value to the short-term traffic flow forecasting. At first, we combine the dual loop speed traps and the cloud computing for significant improvements capability of this detector, provide an efficient processing for the proposed data collection method, provide higher bandwidth to tackle low reception rate problem. After that, by adopting Choquet integral operator on collected data speed after a slot of time, foreseen arrival time and busiest arrival time are introduced as two traffic parameters. Whereas, it is possible to deduce the traffic flow conditions thought the relationship between them. Also, we attempt incorporating these two parameters on the traffic flow forecasting process. To demonstrate the accurateness of the proposal, applied simple linear regression demonstrates high correlation between aggregated busiest arrival time and the traffic flow $(q)$. This correlation proves that the foreseen arrival time could be considered as key parameter for short-term traffic forecasts. Moreover, simulation results indicate the proposed data collection method with Choquet integral is accurateness and closes to the real data compared with weighted average operator. 
The remaining parts of this paper are arranged as follows. The section 2 denotes our proposed cloud-service for short-term traffic flow forecasting. While the section 3, dealt with our proposed real-time data collection method for traffic flow forecasting. Section 4, includes applied linear regression for the aforementioned process. While our conclusion work is given is section 5 .

\section{Cloud based-service for traffic flow forecasting}

The deployment of the cloud computing technology with wireless sensor network (WSN) has the potential to be the most promising service for traffic detection applications. Through this deployment, a significant enhancement of WSN capability on data storage and processing are proven [10]. In this study, we are interesting by dual loop speed detectors to forecast short-term traffic flow, it used as an operated sensor to detect individual vehicle speed. From end to end combining cloud based-service with these detectors, an automatic offset of data can achieved through continues connection to the cloud service. Moreover, we deploy cloud computing technology in term of efficient computing and high bandwidth. The first characteristic aimed to bring more accuracy value to the traffic flow forecasting. Whereas the high bandwidth characteristic is to tackle the problem of low reception rate, insufficient bandwidth and network saturation.

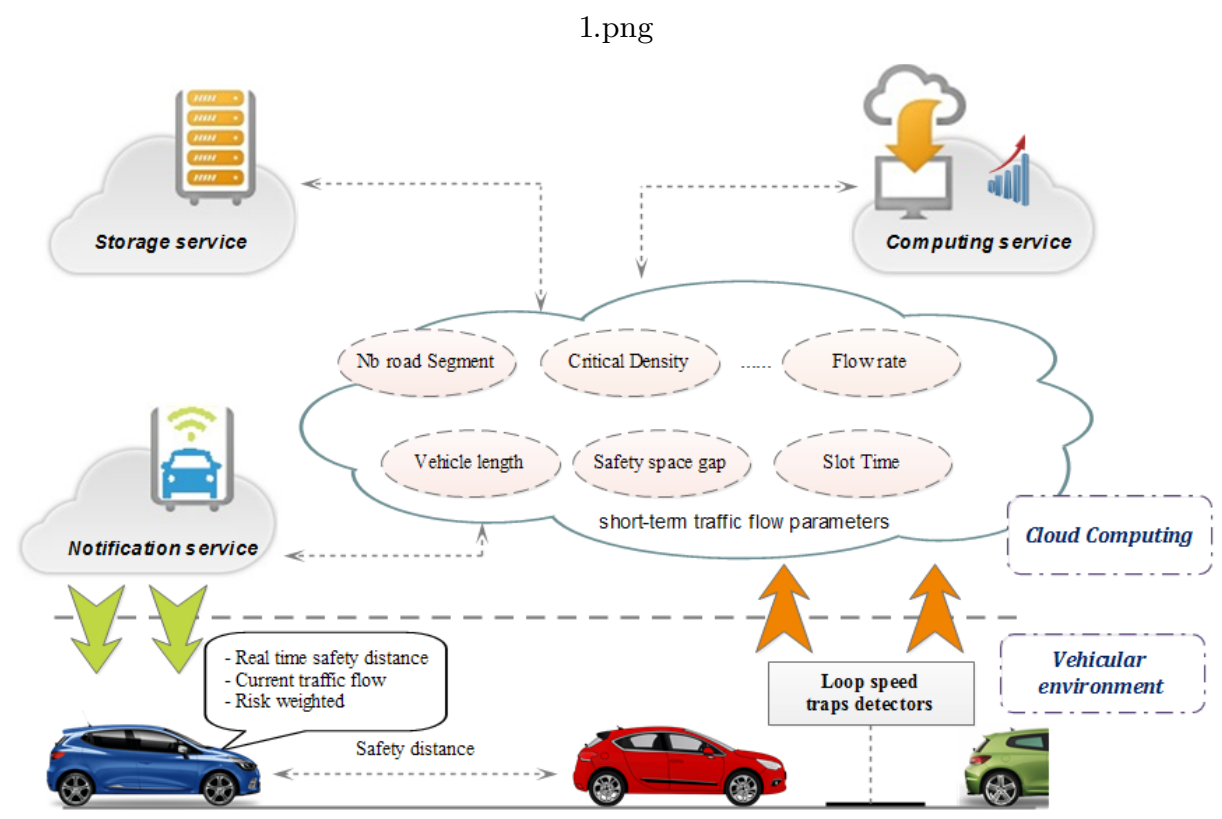

Figure 1. Cloud based-service architecture for short-term traffic forecasting 
The figure 1 outlines our proposed cloud based-service to forecast short-term traffic flow. As key feature, from real time data provided by dual loop speed detectors, the cloud service identify short-term traffic flow parameters such a safety space gap, average speed, critical road segment, entering rate, etc. At this level, three potential cloud based-services dealt with short-term traffic flow parameters: computing service, storage service and notification service. The computing service is usefull for data processing and performing intensive computing on short-time traffic data such for example: runs genetic algorithms for heuristic solutions, forecasting traffic flow using a linear regression as included in the next section. The storage service offers sustainable large storage capacity for real time traffic data and thus, further data analysis and decisions making to the transportation department. Furthermore, the cloud service has two communication interfaces with the vehicular environment. The first interface concern direct communication with dual loop speed detectors. While notification interface enables notifying all vehicles with real time traffic information such as: real time safety distance, current traffic flow and the risk weight.

Through an automatic identification of traffic parameters by the cloud service, our proposed architecture is intended to bring more advantages for emerged modern car applications like fleet management, pedestrians and queue safety. Where data notifications are relevant for all vehicles that are found in the cloud coverage area.

\section{Real-time data collection method for traffic flow forecasting}

By assuming, that drivers on the road has a great interesting to know current traffic flow status $(q)$ as well as the risk which may be subjected during their trips. For this, an installed dual loop speed detectors on road of $3 \mathrm{~km}$ to record observed individual speed counts. Thus, we denote $(S p i)$ to the vehicle speed at loop detector and $(L v)$ to the vehicle length (In this work we consider only the length of the light vehicles). After a slot of time, the proposed method operated microscopic parameters as inputs such as observed vehicle speeds, road length $(d)$ and the vehicle length $(L v)$. On which the main purpose is to forecast an accurate traffic flow from observed speed counts. As key novelty, we introduce two traffic parameters called foreseen arrival time and busiest arrival time, which they are, warrants our intention to predict the traffic flow and highlight its impacts on safe driving.

\subsection{Foreseen arrival time parameter}

Estimated arrival time, is defined as necessary time which must elapse between two consecutive vehicles. To determine its value, a safety distance and the average speed are needed. For more safety, a safety distance $(S d)$ should be determined because it describes required reaction time for a driver to react with a critical situation on the road [3]; this time is accurately close of 2 s. In addition, the 
vehicles must maintain this distance in order to avoid the risk of collision in case of sudden deceleration or sudden stop of ahead vehicles. The Eq.1 in the following set the safety distance measured in meters which is closes to $5 / 9$ of average speed:

$$
S d=A v g \text { Speed } * 5 / 9
$$

After determining safety distance in accordance with the previous equation, the proposed method split the road length into segments. On which each segment length can be identified according to the Eq.2:

$$
\text { SegLengh }=L v+S d
$$

At slot of time j, assume $\mathrm{Spj}=\{S p 1, S p 2, \ldots, S p n\}$ represents a set of observed speed counts by utilizing speed trap detectors, and being aggregated as well. Where $\mu\left(\Delta S p_{(i)}\right)$ embodies a weight of importance which excludes two consecutive speeds since greatest difference speed between them. $C \operatorname{sp}\left(S p_{1}, S p_{2}, \ldots, S p_{n}\right)$ denotes determined busiest speed average at this slot by Choquet integral operator and further satisfying the following equation:

$$
C \operatorname{sp}\left(S p_{1}, S p_{2}, \ldots, S p_{n}\right)=\sum_{i=1}^{n}\left(S p_{(i)}-S p_{(i+1)}\right) \mu\left(\Delta S p_{(i)}\right)
$$

Now, we can compute foreseen arrival time (FArrT) between two vehicles by the following equation:

$$
F A r r T=S e g L e n g h / C s p\left(S p_{1}, S p_{2}, \ldots, S p_{n}\right)
$$

\subsection{Busiest arrival time for Safety Traffic flow}

Safer traffic flow reflects safety importance which is depending on observed traffic conditions in time and space, where it can lead to low-risk driving. For instance, two traffic conditions affect the traffic safety, maintaining safety distance by the vehicles and closer individual vehicle speed to the average speed. Indeed, it is crucial to aggregate observed arrival times between all vehicles for individual segment to busiest arrival time $\Delta t_{(p)}$ for all segments. After that, this busiest time will play an important role in identifying safer traffic flow level. By utilizing Choquet integral operator, compute $\Delta t_{(p)}$ parameter is giving by following equation:

$$
\Delta t_{p}\left(t_{1}, t_{2}, \ldots, t_{n}\right)=\sum_{i=1}^{n}\left(t_{(i)}-t_{(i+1)}\right) \mu\left(\Delta t_{(i)}\right)
$$


Where $t_{1}, t_{2}, \ldots, t_{n}$ presents observed arrival times between pair of vehicles; $\mu\left(\Delta t_{(i)}\right)$ implies a weight that eliminates two consecutive arrival times since greatest difference time between them.

Regardless to the equations (4) and (5), a new real-time traffic conditions can be identified by comparing foreseen arrival time and busiest derived arrival time. In particular, if $\Delta t_{(p)}$ is greater than FArrT that implies subjected vehicles in low risk driving. Otherwise, there is a significant weight of risk could be considered for current traffic flow. In contrast, traffic flow forecasts utilizing weighted operator can be attained through replacing equations (3) and (5) by the equations ( 6 ) and (7) respectively:

$$
\begin{gathered}
\operatorname{AvgSp}\left(S p_{1}, S p_{2}, \ldots, S p_{n}\right)=\sum_{i=1}^{n} S p_{(i)} / N \\
\Delta t_{p}\left(t_{1}, t_{2}, \ldots, t_{n}\right)=\sum_{i=1}^{n} t_{(i)} / N
\end{gathered}
$$

In summary, for an accurate traffic flow forecasts with the proposed method, the correlation between traffic flow $(q)$ and busiest arrival time could be studied. In particular, a simple linear regression is applied in order to demonstrate high correlation between these two parameters. Extended details of applied simple linear regression analysis will be included in the next section.

\section{Linear regression for traffic flow forecasting}

In this section, we attempts to explore the relationship between the busiest arrival time and the traffic flow $(q)$. Furthermore, positive correlation demonstrates a valuable traffic forecasts using the proposed busiest arrival time. To reach this purpose, a simple linear regression analysis was applied to explore the relationship between these two variables according to the following general equation:

$$
Y_{j}=b_{0}+\sum_{i=1}^{n}\left(b_{i} X_{i j}\right)+\epsilon
$$

Where $Y_{j}$ presents the traffic flow counts at slot of time $j ; b_{0}$ denotes a constant; $X_{i j}$ refers to busiest arrival time counts; $\epsilon$ are usually denotes the noise (error); $b_{i}$ indicates the regression coefficient.

Traffic flow forecasting with our proposal was realized using omnet ++ simulator [11]. For accuracy purpose, thanks to the supplied real time traffic data by department of transportation. These data are concern $3 \mathrm{~km}$ of road length and it was recorded using loop speed detectors over $4 \mathrm{~min}$ of time, where the individual vehicle speed and the vehicle arrival times are recorded. With slot time 
of $20 \mathrm{~s}$, the foreseen arrival times of all vehicles are computed and furthermore assimilated for busiest arrival time aggregation. Nevertheless, we consider just one scenario that implements Choquet integral operator in one hand, weighted average operator in the other hand. Forecasting performance of our proposed method with Choquet integral operator is showed in Fig. 2.

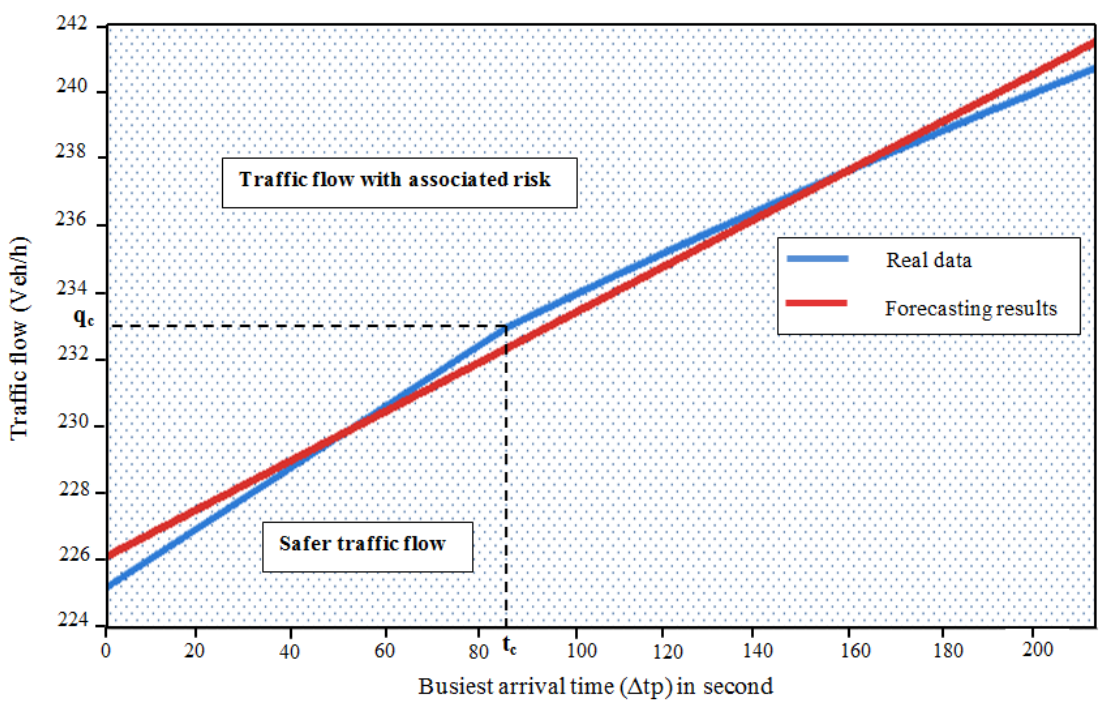

2.png

Figure 2. Forecasting performance of the proposed method with Choquet integral operator.

As we can see, the forecasting results are high closes to the real time data. In addition, with Choquet integral operator, a critical foreseen arrival time $\left(t_{c}\right)$ is corresponding to critical value of traffic flow $\left(q_{c}\right)$. Positively, we can distinct safer traffic flow from traffic flow with likelihood of rear-end collision. This letter take us conclude that the safety distance between the vehicles are not respected while the traffic flow is greater than $q_{c}$. In contrast, the Fig. 3 shows the forecasting performance of our proposed method with weighted average. Which is remarkable is that the forecasting results are weak closes to the real time data comparing with forecasting performance in the Fig. 2. Moreover, the absences of critical point of the traffic flow make the distinction between the safer traffic flow and traffic flow with likelihood of rear-end collision very difficult.

\section{Conclusion}

In this paper, we have proposed a cloud service-based method treats with shortterm traffic flow prediction accuracy. An efficient data collection mechanism is ensured by combining the cloud system and the speed trap detector. In this 


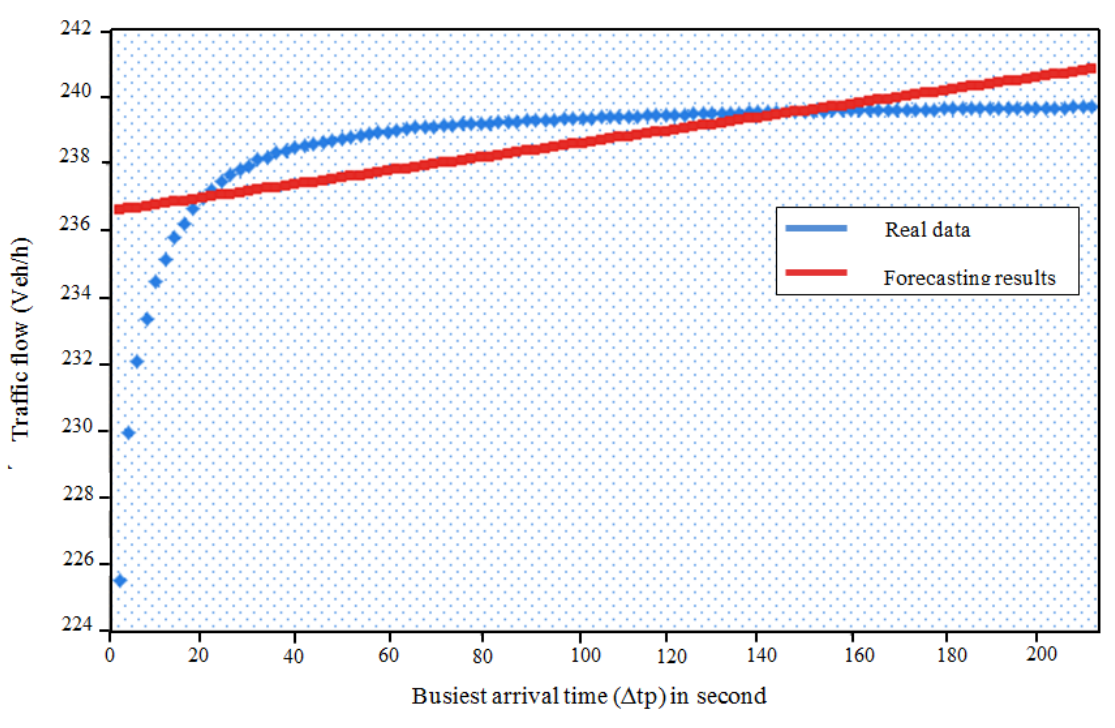

3.png

Figure 3. Forecasting performance of the proposed method with weighted average operator.

way, two traffic parameters were introduced, foreseen arrival time and busiest arrival time. By apply Choquet integral operator, the foreseen arrival times will be aggregated to the busiest arrival time. At the simulation stage, the simple linear regression demonstrates high coloration between busiest arrival time and the traffic flow $(q)$. For more accuracy, Choquet integral operator offers an accurateness value to the traffic flow instead of weighted average operator. This take us concluded that the busiest arrival time is importantly and it has a great impact on the traffic flow prediction. Also, we argue that the Choquet integral utilization for data aggregation is appropriate for safety and future of traffic conditions. While in the future works, we attempt to propose hybrid model for short-term traffic flow estimation by incorporating real-time data and historical data.

Acknowledgments this work is subscribed in the context of the thematic research project entitled "Integrated Road Traffic in Algeria", our objective is to develop VANETs applications that are "suspected" of spatiotemporal context, so to generalize the exchange of V2V information's (Vehicle-to-Vehicle), V2I information's (Vehicle-to-Infrastructure) and even V2X, and support all communication types (radio, internet, etc.) in their activities 


\section{References}

1. Bachmann, C., Abdulhai, B., Roorda, M.J., Moshiri, B.: A comparative assessment of multi-sensor data fusion techniques for freeway traffic speed estimation using microsimulation modeling. Transportation research part C: emerging technologies 26, 33-48 (2013)

2. Bai, C., Peng, Z.R., Lu, Q.C., Sun, J.: Dynamic bus travel time prediction models on road with multiple bus routes. Computational intelligence and neuroscience $2015,63(2015)$

3. amended by Decree No: Article R412-12. Tech. rep.

4. Feng, Y., Head, K.L., Khoshmagham, S., Zamanipour, M.: A real-time adaptive signal control in a connected vehicle environment. Transportation Research Part C: Emerging Technologies 55, 460-473 (2015)

5. Ji, H., Xu, A., Sui, X., Li, L.: The applied research of kalman in the dynamic travel time prediction. In: Geoinformatics, 2010 18th International Conference on. pp. 1-5. IEEE (2010)

6. Lindveld, C.D., Thijs, R., Bovy, P., Van der Zijpp, N.: Evaluation of online travel time estimators and predictors. Transportation Research Record: Journal of the Transportation Research Board (1719), 45-53 (2000)

7. Moretti, F., Pizzuti, S., Panzieri, S., Annunziato, M.: Urban traffic flow forecasting through statistical and neural network bagging ensemble hybrid modeling. Neurocomputing 167, 3-7 (2015)

8. Patnaik, J., Chien, S., Bladikas, A.: Estimation of bus arrival times using apc data. Journal of public transportation 7(1), 1 (2004)

9. Qian, J., Eglese, R.: Fuel emissions optimization in vehicle routing problems with time-varying speeds. European Journal of Operational Research 248(3), 840-848 (2016)

10. Savas, O., Jin, G., Deng, J.: Trust management in cloud-integrated wireless sensor networks. In: Collaboration Technologies and Systems (CTS), 2013 International Conference on. pp. 334-341. IEEE (2013)

11. Varga, A., et al.: The omnet++ discrete event simulation system. In: Proceedings of the European simulation multiconference (ESMâĂŹ2001). vol. 9, p. 65. sn (2001)

12. Williams, B., Durvasula, P., Brown, D.: Urban freeway traffic flow prediction: application of seasonal autoregressive integrated moving average and exponential smoothing models. Transportation Research Record: Journal of the Transportation Research Board (1644), 132-141 (1998)

13. Yasmin, S., Eluru, N., Lee, J., Abdel-Aty, M.A.: An ordered fractional split approach for aggregate injury severity modeling (2015)

14. Zhong, J.t., Ling, S.: Key factors of k-nearest neighbours nonparametric regression in short-time traffic flow forecasting. In: Proceedings of the 21st International Conference on Industrial Engineering and Engineering Management 2014. pp. 9-12. Springer (2015) 